\title{
Computational Aspects of Model Acquisition and Join Geometry for the Virtual Reconstruction of the Atrahasis Cuneiform Tablet
}

\author{
Tim Collins*, Sandra Woolley ${ }^{\dagger}$, Erlend Gehlken ${ }^{\ddagger}$ and Eugene Ch’ng ${ }^{\S}$ \\ *Manchester Metropolitan University, UK \\ Email: T.Collins@mmu.ac.uk \\ ${ }^{\dagger}$ Keele University, UK \\ Email: s.i.woolley@keele.ac.uk \\ $\ddagger$ Universität Frankfurt, Germany \\ Email: Gehlken@em.uni-frankfurt.de \\ $\S$ University of Nottingham Ningbo China \\ Email: eugene.chng@nottingham.edu.cn
}

\begin{abstract}
The epic of Atrahasis is one of the most famous pieces of ancient Mesopotamian literature. The account has survived millennia on sets of clay tablets inscribed with cuneiform script; a sophisticated early writing system comprising signs formed from wedge-shaped impressions. The third tablet belonging to one of the most complete copies of the Atrahasis epic is broken. For over fifty years, one fragment, held in Geneva, was believed to join with another held in London. However, due to their $1000 \mathrm{~km}$ separation, the join had never been physically tested. This paper contributes a technological account of the successful virtual joining of the fragments [1]; the first ever longdistance virtual join of its type.
\end{abstract}

\section{INTRODUCTION}

The Atrahasis epic is an important piece of ancient Mesopotamian literature. It describes a creation myth, a great flood and the building of an ark and it pre-dates, by centuries, similar accounts in the Book of Genesis [2]. The epic is recorded in several surviving copies of clay cuneiform tablets which are largely fragmented. The 'Old Babylonian' Atrahasis tablets, written by the scribe Ipiq-Aya in the seventeenth century $\mathrm{BCE}$, form the most famous Akkadian copy of the epic. Fragments of these tablets are currently held in collections in London, New Haven, New York and Geneva [3]. The third tablet in the set, describing the flood and the building of the ark, is broken and believed to be separated between two museums. The larger fragment of this tablet (museum number BM 78942+78971+80385, known as $C_{1}$ ) is held in the British Museum in London whilst the other (museum number MAH 16064, known as $C_{2}$ ) is held at the Musée d'Art et d'Histoire in Geneva. Being separated by approximately $1000 \mathrm{~km}$, this suspected join had never been verified prior to the virtual reconstruction [1].

As part of the Virtual Cuneiform Tablet Reconstruction Project, [4], [5] virtual reconstructions have been reported for typically small form factor tablet fragments collocated within individual collections [6], [7]. This paper provides an account of the computational aspects of model acquisition and join geometry for the virtual reconstruction of fragments of a different form factor, in different collections, located in different countries. The join achieved, first reported in [1], is the first such long-distance join of its type.

\section{ACQUISITION}

\section{A. The Virtual Cuneiform Tablet Reconstruction Project Ac- quisition System}

A significant challenge in the virtual reconstruction of fragmented artefacts is the acquisition of the virtual fragments themselves [8]. Conventional laser scanners and structured light scanners are costly and not easily portable. In addition, the scanning process can be labour intensive, requiring training and skills in order to acquire partial 3D models from multiple viewpoints before manually 'stitching' the parts together to form a complete 3D mesh.

As part of the Virtual Cuneiform Tablet Reconstruction project, a low-cost, portable acquisition system based on photogrammetric processing has been developed. The system consists of a camera and turntable synchronised to a laptop computer to automatically capture sequences of photographstaken from obverse, reverse and edge views. A smartphone with an integrated camera can be used in place of the computer and camera for a very low-cost and portable acquisition system.

In multi-viewpoint photogrammetric acquisition [9], sets of photographs are obtained by either moving around the object taking pictures from multiple viewpoints, or by fixing the camera and rotating the object on a turntable [10]. The turntable approach has the advantage of speed and ease of use although care must be taken to maintain consistent lighting conditions and to eliminate background features by the use of a matt, monochrome tabletop cover.

A block diagram of the rotary electronic control hardware is shown in figure 1.The microcontroller receives instructions from either a computer via a USB serial link or a smartphone 


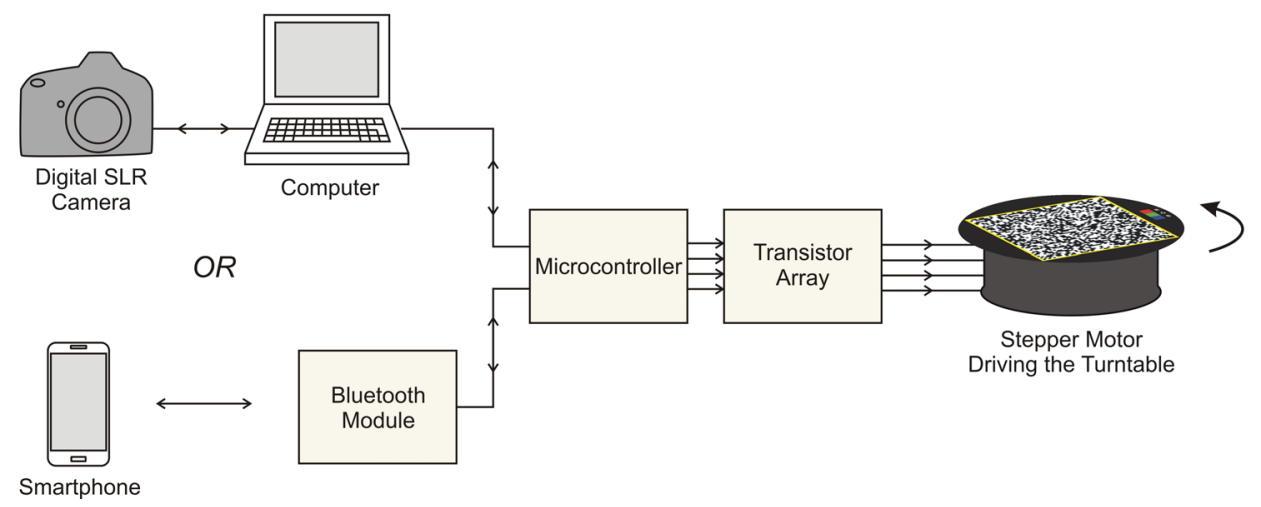

Fig. 1. Block diagram of the hardware components of the rotary acquisition system.

via a Bluetooth receiver module. Application software running on either the computer or smartphone synchronises the turntable motion and the camera trigger.

The photogrammetric reconstruction processing follows the well-established workflow of feature extraction, camera parameter estimation, dense point-cloud reconstruction, meshing then texturing.

Without a known datum, the scale of a photogrammetrically acquired model is arbitrary [11]. A conventional solution is to include additional coded targets and/or scale bars in the image scene. In our system, a pseudo-random calibration pattern adhered to the top surface of the turntable is used for the automated calibration of the reconstructed 3D model.

This system has been tested with laboratory-fabricated synthetic artefacts as well as with approximately 100 cuneiform tablet fragments from the Ur collection currently housed on study loan at the British Museum [7]. An example of the models obtained from two Ur fragments, that were known to join, is shown in figure 2 and demonstrates that the accuracy and precision of the system is sufficient to verify matching joins between fragments.

\section{B. Acquiring the Atrahasis Fragments}

The acquisition system was designed with typical cuneiform tablet dimensions in mind. In a survey of 8000 catalogued tablets extracted from the Cuneiform Digital Library Initiative (CDLI) database [12], the average width and length were found to be $43 \mathrm{~mm}$ and $51 \mathrm{~mm}$ respectively [13].

Fragment $C_{2}$ is a similar form-factor to the typical tablet fragments and was acquired using the digital camera version of the system without modification. Fragment $C_{1}$ is substantially larger than most cuneiform tablets and over twice the size of any fragment previously acquired with the system. Large cuneiform tablets such as this pose unusual challenges in their acquisition. Fragment $C_{1}$ is sufficiently large and fragile that one would, ideally, wish to photograph it in a static setting, obtaining structure from motion by moving the camera in the fashion commonly employed for statues or monuments. However, cuneiform fragments were created with handling in mind and have important information on all surfaces so

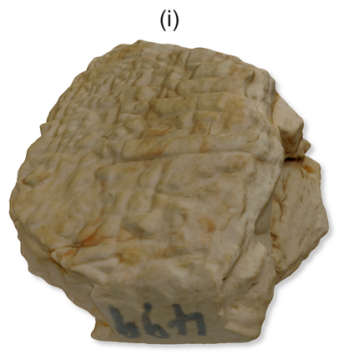

(ii)

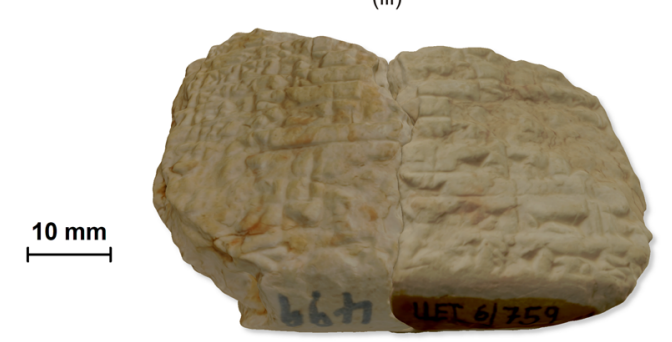

Fig. 2. Visualizations of a pair of cuneiform tablet fragments, (i) UET 6/748 and (ii) UET 6/759, automatically joined in virtual form with the result shown in (iii).

viewpoints from the obverse, reverse and, ideally, the edges are desirable.

Due to the unusual size, weight and fragility of $C_{1}$, some compromise was necessary. Although obverse and reverse viewpoints were straightforward using our rotary acquisition system, balancing the tablet on its side to achieve edge-up viewpoints was not possible. Tablet $C_{1}$ is actually a composite of four fragments that have been glued together. Extreme care must be taken when handling such large, fragile tablets and it was not safe to attempt to prop it on edge. The 3D model for this tablet had to be reconstructed based solely on obverse and reverse viewpoints. The substantial thickness of the tablet (approximately 40mm - over twice the normal thickness) helped and, with adjustments to the camera angle and lighting, sufficiently detailed views of the edges were photographed and the 3D model reconstructed.

Since tablet $C_{1}$ is too large to fit within the 


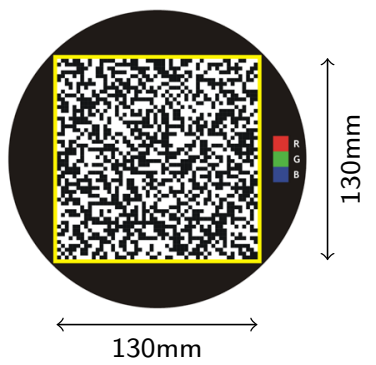

(ii)

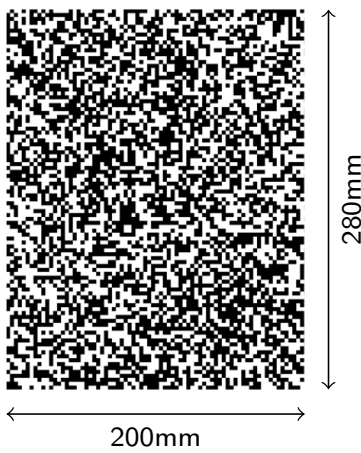

Fig. 3. Calibration plates: (i) The existing $130 \mathrm{~mm} \times 130 \mathrm{~mm}$ calibration pattern as used in Geneva for fragment $C_{2}$, (ii) the custom $200 \mathrm{~mm} \times 280 \mathrm{~mm}$ calibration pattern used in London for fragment $C_{1}$. In both cases, the patterns are made from a grid of $2.5 \mathrm{~mm} \times 2.5 \mathrm{~mm}$ pseudo-randomly coloured squares.

$130 \mathrm{~mm} \times 130 \mathrm{~mm}$ area of our usual calibration pattern, a custom $200 \mathrm{~mm} \times 280 \mathrm{~mm}$ calibration plate was specially made for the purpose (figure 3). This necessitated a new set of synthetic reference photographs to be created for the calibration process.

Although the tablet fragment $C_{1}$ is significantly larger than most cuneiform tablets, there were no fundamental problems in applying the same photogrammetric processing techniques. Photogrammetric reconstruction works at any scale but it must be noted that the resolution and precision of the process scales with the size of the object. An experimental comparison of our acquisition system with a high resolution 3D scanner had been previously conducted. Using test artefacts approximately $25 \mathrm{~mm}$ wide, the root-mean-squared error of the surface mesh points was found to be just under $50 \mu \mathrm{m}$. Tablet $C_{1}$ is approximately ten times larger than the test artefacts meaning a precision of the order of $500 \mu \mathrm{m}$ was to be expected in this case. Although this is not sufficient for analysis of the text on the tablet, it has proven sufficient for testing and verifying matches.

In spite of the challenges involved in acquiring the fragment models, the quality of the resulting models appeared to be sufficiently adequate for a joining attempt to be made.

\section{JOINING}

\section{A. Automated Joining of Fragmented Cuneiform Tablets}

The overall project aim of virtual cuneiform tablet reconstruction [4] has been investigated using an online collaborative crowd-sourced approach [14] as well as an automated joining process [6]. The automated process was used for the Atrahasis fragments because it reliably ensures that the optimal matching orientation is found and also yields statistics indicating the goodness of the fit.

The first stage of the matching process is to determine the minimum volume oriented bounding box of each fragment. In many cases, approximate alignment of inscribed obverse and reverse sides of the fragments is achieved for opposing pairs of box faces.

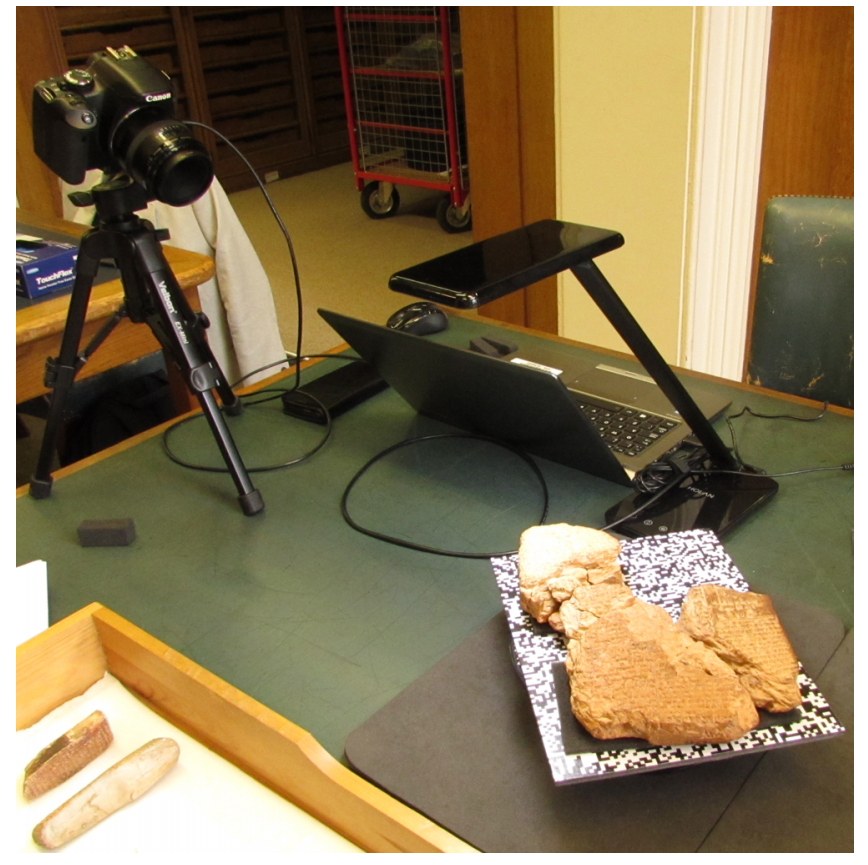

Fig. 4. Tablet $C_{1}$ prior to acquisition at the British Museum. The turntable is almost completely hidden underneath the $200 \mathrm{~mm} \times 280 \mathrm{~mm}$ calibration plate made especially for this tablet.

During the joining process, pairs of box-sides are manipulated and tested using an iterative optimisation algorithm. This optimisation is based on the minimisation of a costfunction derived from the distances between opposing points on the tablet fragment surfaces. These distances are obtained from depth-maps efficiently calculated using GPU (Graphical Processing Unit) optimised processing. A piecewise linear mapping function is used to calculate the cost of candidate orientations and displacements. This mapping function helps to ensure that the correct optimal orientation is found even when the two fragment faces are incomplete and eroded [6]. The best result from these matching attempts is saved and presented as the result.

\section{B. Joining the Atrahasis Fragments}

The automated cuneiform joining algorithm was used without modification to join the virtual Atrahasis fragments. The fragments illustrated in figure 5, along with their bounding boxes, are shown individually and, on the right, together in the pose determined to minimise the cost function. The rotations and translations required are reported and the rotation can be seen by comparing the bounding box orientations. The match appears good with the tablet edge and the inscribed surfaces lining up well. To make a quantitative assessment of the join quality, examination of the corresponding depth maps shown in figure 6 is needed.

The summed depth map is formed by taking the sum of the depth maps of the individual fragments and subtracting the minimum value. This is equivalent to moving the two fragments together until they are just in contact and then measuring the inter-surface distances. Black areas on the 

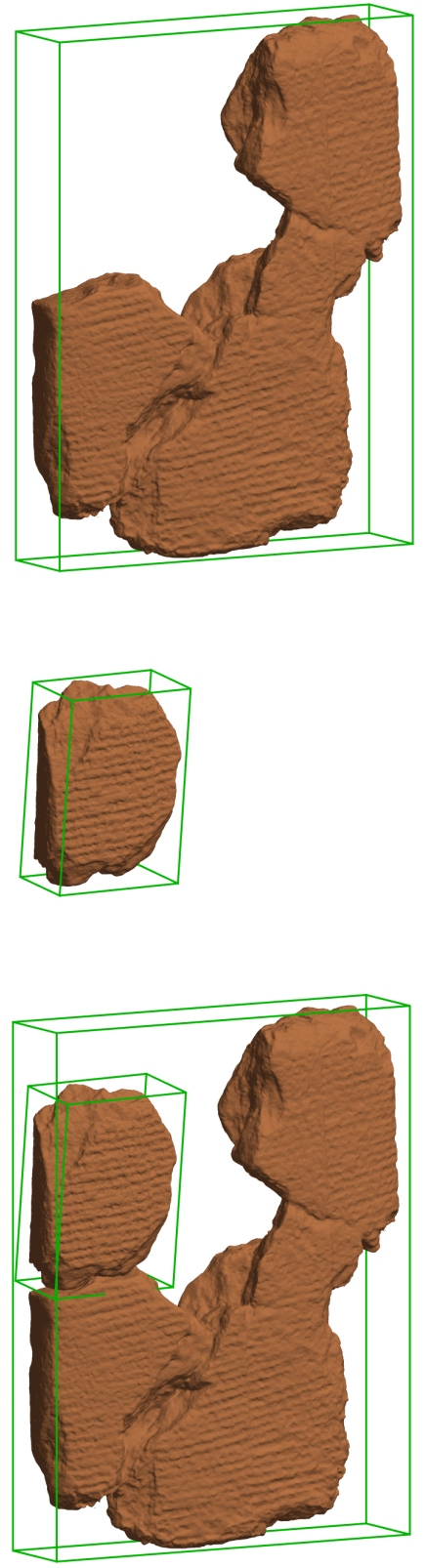

Fig. 5. Results of applying the automated cuneiform matching algorithm to the Atrahasis fragments and their bounding boxes: (top) fragment $C_{1}$, (centre) fragment $C_{2}$, (bottom) fragments $C_{1}$ and $C_{2}$ together.

summed map correspond to zero depth and indicate perfect joining. Within the majority of the map, this does, indeed, appear to be the case. Larger distances around the edges were expected given the levels of erosion clearly visible on the tablets. Within the joining surface, however, the depth is close to zero.

Figure 7 shows a histogram of the depths in the summed depth map. The majority of depths lie between 0 and $2 \mathrm{~mm}$. Depths beyond $2 \mathrm{~mm}$ are less frequent and correspond to the area around the edges of the tablet where depth is greater
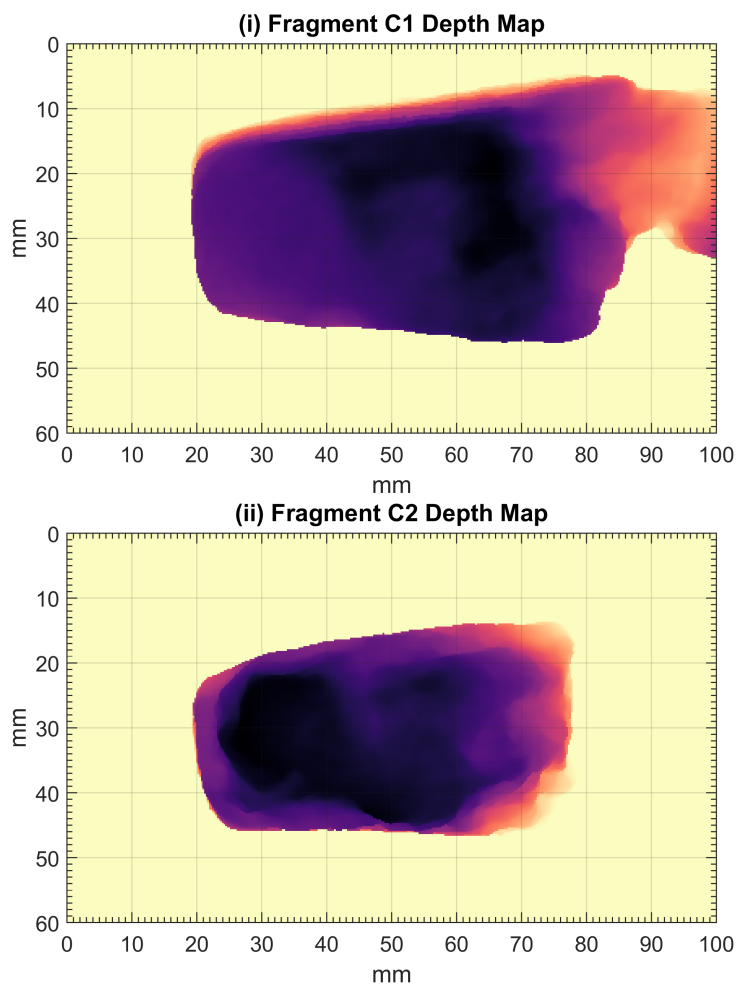

(iii) Summed Depth Maps

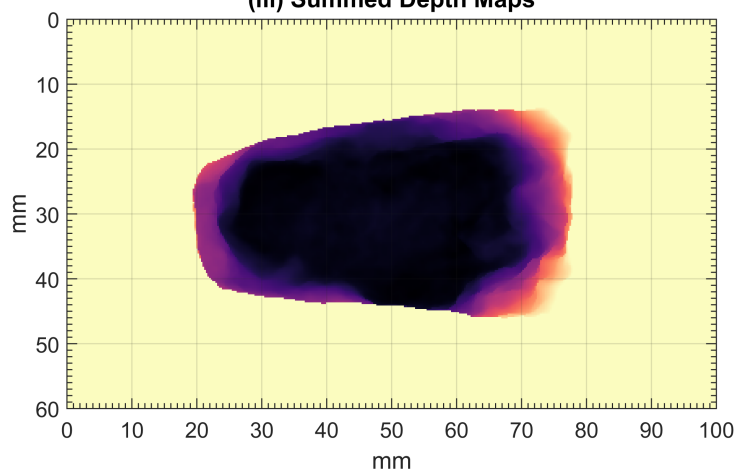

(iv) Joining Area

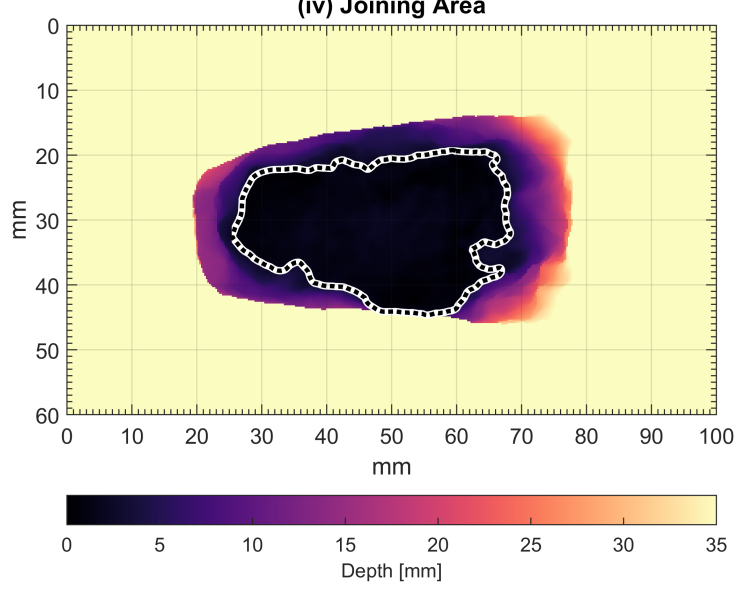

Fig. 6. Depth maps of (i) fragment $C_{1}$, (ii) fragment $C_{2}$, (iii) summed depth maps, (iv) summed depth maps with a depth contour of $2 \mathrm{~mm}$ - used for evaluation of the join's goodness of fit. 
due to erosion. To analyse the goodness of fit of the joining area only, a second histogram in figure 7 shows the frequency of depths using only the pixels inside the contour indicated by the dashed outline in figure 6(iv). The contour is defined by a boundary outside of which all depths are greater than $2 \mathrm{~mm}$. Some isolated values greater than $2 \mathrm{~mm}$ exist within this boundary and cause the small 'tail' seen in the distribution.

There are no negative depths in either histogram because the joining algorithm prohibits any overlap between the virtual fragments. This restriction is based on an assumption that the source data is perfect which we know is not the case. Analysis of the depth distribution within the joining area shows that if a small overlap is permitted and the fragments brought together by a further $1.13 \mathrm{~mm}$ (the mean of the depths within the joining area), the standard deviation of the depths within the join will be $0.47 \mathrm{~mm}$. This figure corresponds to the estimated expected acquisition precision of the $3 \mathrm{D}$ model of fragment $C_{1}$ and, as such, we would not expect to see better results than this - even for an absolutely perfect join.

Given that the solution discovered by the automatic algorithm is consistent with the position and orientation opined by Assyriological scholars and that the statistical analysis of the depth information outlined above indicates a join that is perfect to within the tolerance of the acquisition system, we can conclude that the fragments do, indeed, join.

\section{Results AND CONCLUSions}

Although the automated matching algorithm had no prior knowledge of the suspected join between $C_{1}$ and $C_{2}$, an optimal solution was achieved. This solution is consistent with the position and orientation opined by Assyriological scholars. Despite edge erosion around most of the outer rim of the two broken surfaces, the large region across the whole of the interior demonstrated an excellent interlocking fit for the two fragments that has provided real and robust evidence of the join. The standard deviation of the depths between the joining surfaces has been calculated to be $0.47 \mathrm{~mm}$ - almost exactly the expected precision of the acquisition system for the larger tablet, $C_{1}$. This numerical evidence is backed up by the subjectively correct positioning of the tablets in their joined orientation and indicates a positive join with a high degree of confidence.

Virtual cuneiform tablet reconstruction has previously been demonstrated for automatically identifying joins within a collection [7]. This join [1] has demonstrated that there need be no geographical limitations and joins can be found between collections in different parts of the world. Fragments $C_{1}$ and $\mathrm{C}_{2}$ of the Old-Babylonian Atrahasis tablet had been believed to match for over fifty years. As reported in [1], this match can now be confirmed despite the physical fragments remaining separated by $1000 \mathrm{~km}$ throughout the process.

\section{ACKNOWLEDGEMENTS}

The authors would like to thank the Trustees of the British Museum for permission to access the scanned fragments BM $78942+78971+80385$ and would also like to thank Dr

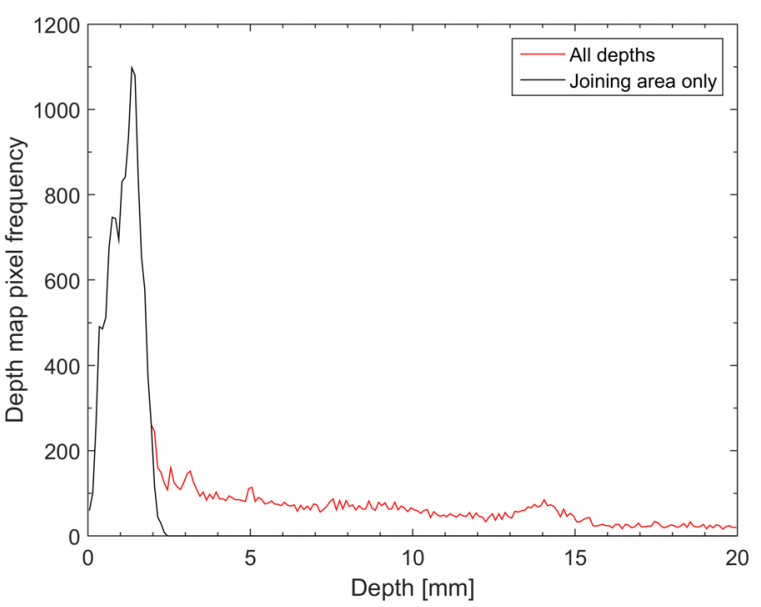

Fig. 7. Depth histograms for the whole of the fragment edges and for just the joining area (as indicated on figure 6(iv) by the dashed contour).

Jonathan Taylor whose assistance made this work possible. The authors would also like to thank Mrs Béatrice Blandin, Geneva, Musée d'Art et d'Histoire, for the permission to scan the fragment MAH 16064.

The cuneiform tablet fragments presented in figure 2 are held on study loan at the British Museum. The authors would like to thank the Trustees of the British Museum for permission to access the fragments and would also like to thank Birger Helgestad, Mathilde Touillon-Ricci and Jonathan Taylor for their assistance.

\section{REFERENCES}

[1] E. Gehlken, T. Collins, S. Woolley, E. Ch'ng, L. Hanes, A. Lewis, and L. Munoz, "Searching the Past in the Future - Joining Cuneiform Tablet Fragments in Virtual Collections," in 63rd Rencontre Assyriologique Internationale, 2017.

[2] W. G. Lambert and A. Millard, Atra-Hasis: The Babylonian story of the Flood. Eisenbrauns, 1969.

[3] I. Finkel, The Ark Before Noah: decoding the story of the flood. Hachette UK, 2014.

[4] VCTR, "Virtual Cuneiform Tablet Reconstruction Project," 2016. [Online]. Available: http://virtualcuneiform.org/

[5] E. Ch'ng, S. I. Woolley, E. Gehlken, A. Lewis, L. H. Munoz, and T. Collins, "The development of a collaborative virtual environment for 3D reconstruction of cuneiform tablets," in Virtual Systems Multimedia (VSMM), 2014 International Conference on, Dec 2014, pp. 35-42.

[6] T. Collins, S. I. Woolley, A. Lewis, L. H. Munoz, E. Ch'ng, and E. Gehlken, "Computer-assisted reconstruction of virtual fragmented cuneiform tablets," in Virtual Systems Multimedia (VSMM), 2014 International Conference on, Dec 2014, pp. 70-77.

[7] E. Gehlken, T. Collins, S. Woolley, L. Hanes, A. Lewis, L. Munoz, and E. Ch'ng, "From Uruk to Ur: Automated matching of virtual tablet fragments," in 62nd Rencontre Assyriologique Internationale, 2016.

[8] A. R. Willis and D. B. Cooper, "Computational reconstruction of ancient artifacts," IEEE Signal Processing Magazine, vol. 25, no. 4, pp. 65-83, 2008.

[9] P. Bourke, "Automated 3D model reconstruction from photographs," in Virtual Systems Multimedia (VSMM), 2014 International Conference on, Dec 2014, pp. 21-24.

[10] S. T. Porter, M. Roussel, and M. Soressi, "A simple photogrammetry rig for the reliable creation of 3D artifact models in the field," Advances in Archaeological Practice, vol. 4, no. 1, pp. 71-86, 2016. 
[11] A. H. Ahmadabadian, S. Robson, J. Boehm, M. Shortis, K. Wenzel, and D. Fritsch, "A comparison of dense matching algorithms for scaled surface reconstruction using stereo camera rigs," ISPRS Journal of Photogrammetry and Remote Sensing, vol. 78, pp. 157 - 167, 2013.

[12] CDLI, "Cuneiform Digital Library Initiative," 2016. [Online]. Available: http://cdli.ucla.edu/

[13] A. Lewis and E. Ch'ng, "A photogrammetric analysis of cuneiform tablets for the purpose of digital reconstruction," International Journal of Heritage in the Digital Era, vol. 1, no. 1, pp. 49-53, 2012.

[14] S. Woolley, E. Ch'ng, L. Hernandez-Munoz, E. Gehlken, T. Collins, D. Nash, and L. Hanes, "A collaborative artefact reconstruction environment," in Proceedings of the British HCI Conference, July 2017. 